\title{
SOFT FLEXION SENSORS INTEGRATING STRECHABLE METAL CONDUCTORS ON A SILICONE SUBSTRATE FOR SMART GLOVE APPLICATIONS
}

\author{
Hadrien O. Michaud, Joan Teixidor, and Stéphanie P. Lacour* \\ Laboratory for Soft Bioelectronic Interfaces (LSBI), École Polytechnique Fédérale de Lausanne \\ (EPFL), Lausanne, Switzerland
}

\begin{abstract}
We design and implement a sensory skin that monitors in real time finger flexure (three sensors per finger) of a user's hand. Compared to current technologies, the electronic skin is made entirely of stretchable materials integrating silicone rubber, low resistivity liquid metal interconnects and high strain sensitivity, microstructured thin metal films. Microfabrication of the sensors combines traditional thin film process and additive manufacturing techniques. We incorporate the skin on a textile glove and demonstrate its function as an interface for finger motion and posture detection using a robotic test platform.
\end{abstract}

\section{INTRODUCTION}

Data gloves are sensor systems capable of encoding and reconstructing the posture and the movement of the human hand. Such devices were initially developed for animation and machine interaction purposes but they are now gaining attention for health monitoring and rehabilitation applications [1]. Integrating multiple sensors based on thin and soft materials, such as textile, in order to monitor joints position with minimal motion impediment is a desirable feature that has been recently investigated [2].

Electronic skins incorporating multiple functionalities on ultra-compliant substrates are a promising research area for smart gloves. Recent advances demonstrated sensing of multiple stimuli - including movement, pressure or temperature - via thin and soft systems that can be worn as a second skin by human and robots [3].

As far as movement or strain sensing is concerned, strain gauges appear as a relevant solution since they can be easily interfaced with a wide variety of off-the-shelf micro-electronic components and do not require multilayer architecture or shielding against electromagnetic noise sources. In addition, they are insensitive to other stimuli such as normal pressure and can be temperature compensated.

Soft piezoresisitive composites based on quantum tunneling and percolation between conductive micronanoparticles trapped in a polymer matrix have been extensively reported as potential strain sensing elements for artificial skin with application to human motion detection [4]. However, these material assemblies are prone to creep. It results in a large viscoelastic response to tensile and compressive strains, hindering the dynamic response of the sensors due to overshoot after unloading and large relaxation time $[5,6]$.

Liquid metal microstructures are another popular solution for soft strain sensors. Hammond et al. demonstrated microchannels filled with a liquid metal alloy as strain gauges embedded in a glove-like system for monitoring human finger flexion [7]. However, the need to use rigid, strain insensitive wires as interconnects in order to detect locally the change in resistance of the liquid metal gauges may limit this approach. Moreover, the interface between soft sensors and hard wires is reported as a cause of failure for the system due to leaks and delamination.

In this work, we propose a combination of gold thin films deposited on a soft rubber substrate [8] as strain sensing elements with directly plotted gallium and indium eutectic alloy (EGaIn) micro-wires as interconnects [9]. We assembled three flexion sensors into a thin $(<0.5 \mathrm{~mm})$ and soft silicone membrane. The system was mounted on a humanoid hand and we monitored its static and dynamic performance. The iCub robot was chosen as the test platform since its hand mimics the kinematic of the human fingers and embeds its own commercial rotation sensors [10].

\section{CONCEPT AND DESIGN}

\section{Sensing mechanism and material}

In our implementation, the sensors were integrated in an elastomer membrane placed in tight contact with the dorsal side of the finger. Let's consider an articulated finger with three cylindrical joints (metacarpophalangeal

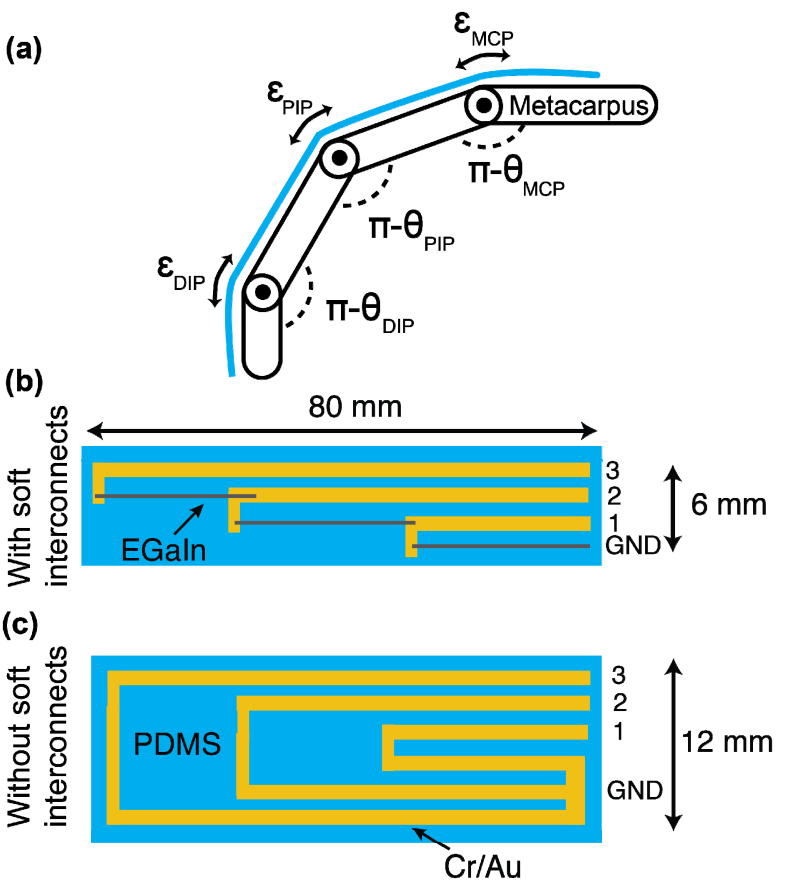

Figure 1: Design and principle of the soft flexion sensing strip with soft interconnects for humanoid fingers. 
MCP, proximal interphalangeal PIP, and distal interphalangeal DIP) of radius $r$ and rigid phalanxes. When a joint of the finger rotates by an angle $\theta$, the length of the dorsal part the finger increases by $\theta r$. It results in a tensile strain $\varepsilon$ across the surface of the soft membrane located on top of the joint (Figure 1a). In order to transduce this mechanical stimulus, we chose to form strain gauges from patterns of stretchable gold thin films [8]. Such films can withstand large deformations imposed by the finger's motion. Strain accommodation mechanism results from microscopic rearrangement of interconnected gold ligaments. The resulting resistance change (hundreds to thousands of Ohms depending on initial resistance $\mathrm{R}_{0}$ ) is essentially linear for tensile strain regimes up to $15 \%$, with gauge factors of the order of 2 to 5.

\section{Design of sensors and soft interconnects}

Soft liquid metal micro-wires embedded in silicone have small resistance at rest (less than $1 \Omega$ for centimeterlong wires) and their resistance increases by less than $150 \%$ for strains up to $50 \%$ [11]. These characteristics were ideal for building a stretchable interconnects network. Three gold-film strain gauges with graded lengths aligned with the middle of the phalanxes of the robotic finger were interconnected as shown Figure $1 b$. The staggered EGaIn interconnect design was laid down because of a limitation from our printing set-up (which restricted to $35 \mathrm{~mm}$ the maximum length of a printed wire). Figure $1 \mathrm{c}$ presents an equivalent design using only stretchable thin gold films. Although such pattern could have been produced in a single evaporation step, it would have had a larger footprint (discarding it for mounting on the $\mathrm{iCub}$ finger) and the implementation of the corresponding shadow mask would have been challenging (thin polyimide features). Furthermore, the liquid metal interconnects provided a fully elastic wire-sensor interface thereby avoiding interfacing mechanically soft sensors with hard wiring and preventing mechanical stress concentration at the soft-hard junctions and failure of the sensory skin.

\section{Equivalent electrical circuit}

Figure 2 presents the equivalent electrical circuit of the soft sensing system. For each gauge $\mathrm{j}, R_{j}>>\Sigma R_{\text {int }, k}$ at rest. When the finger flexed, we had $\Delta R_{j}>>\Delta R_{\text {int }, k}$, so the sensors could be addressed independently through the interconnection network.

\section{FABRICATION METHODS}

The fabrication process flow for the soft metal sensing strips is detailed Figure 3. Polydimethylsiloxane (PDMS, Sylgard 184, Dow Corning) prepolymer and curing agent were mixed in a 10:1 ratio and spun at 500 $\mathrm{RPM}$ on a $100 \mathrm{~mm}$ silicon wafer and cured at $80^{\circ} \mathrm{C}$ for

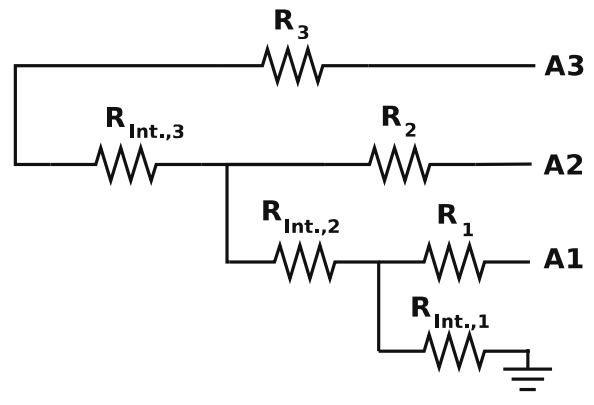

Figure 2: Equivalent electrical circuit of the soft integrated flexion sensing strip.

two hours. $5 / 25 \mathrm{~nm} \mathrm{Cr} / \mathrm{Au}$ thin films were thermally evaporated through a polyimide shadow mask to define the strain sensing patterns. Next, a syringe was loaded with the EGaIn alloy (Sigma Aldrich) and attached to an X-Y-Z stage (GIX microplotter II, Sonoplot) via a custom holder. The syringe's tip inner diameter was $360 \mu \mathrm{m}$. A controlled pressure (approx. 0.02 bar) was applied inside the syringe to force the EGaIn to reach the tip's end without forming a droplet. The tip was lowered about 70 $\mu \mathrm{m}$ above the PDMS layer in order to form a liquid metal meniscus between the tip and the substrate. The stage then followed predefined 2D patterns in the XY plane while pressure was maintained to plot the soft liquid metal interconnects.

Next, solid wires were mounted at the proximal extremity of the strip, connected to soft interconnects or strain gauges using small liquid metal droplets, and secured using silicone sealant (Dow Corning 734).

Finally, a PDMS encapsulation layer was spin-coated at 250 RPM. The final thickness of the strips was below $500 \mu \mathrm{m}$. Figure 4 provides detailed pictures of the soft materials assembly. EGaIn microwires had a semicircular cross-section and their typical diameter was 250 $\mu \mathrm{m}$ (Figure 4a).

We observed the micro-cracked structure in the gold film on PDMS that enabled accommodation of large mechanical deformation and finite electrical resistance (Figure 4b). Gallium and gold formed an electrically conductive alloy that was visible within a few hundred microns around the interconnection area (Figure 4c) [12].

Overall, the skin fabrication process only required four steps. Designs may be easily customized since interconnects were patterned using an additive, mask-less and mold-less process.

\section{RESULTS AND DISCUSION}

\section{Single sensor characterization on the $\mathrm{iCub}$ hand}

After microfabrication, the strip was attached to a textile glove using EcoFlex (Smooth-On) as a curable adhesive. When the robot was wearing the sensorized glove, we observed it could rotate normally all finger's joints and open and close completely its hand (Figure 5).
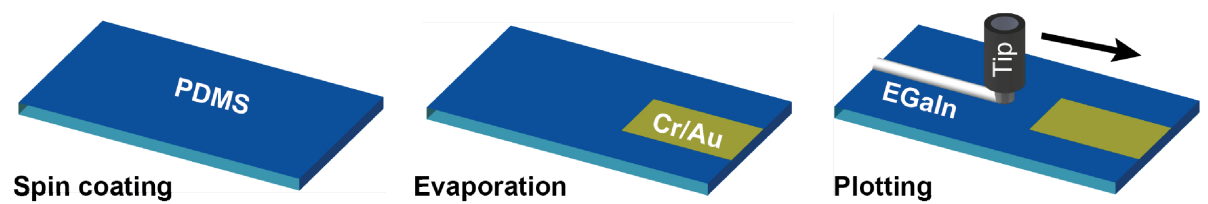

Figure 3: Process flow for the fabrication of a soft metal flexion sensor.

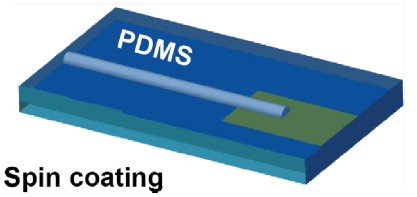




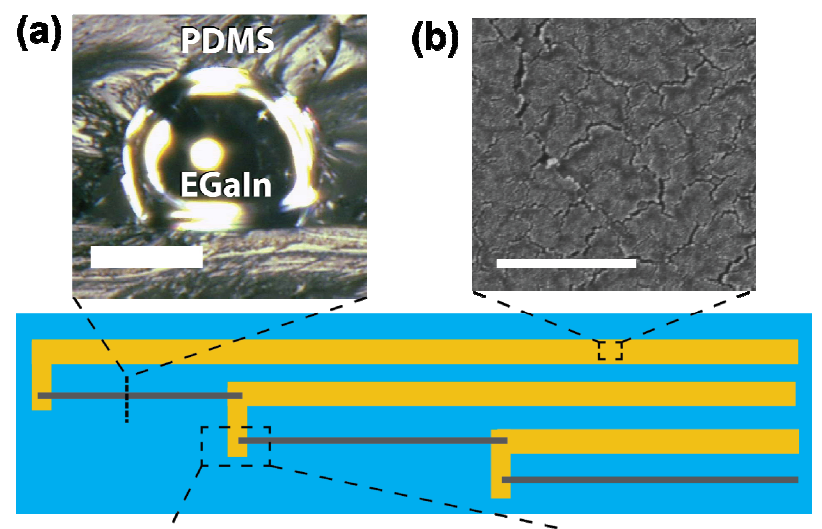

(c)

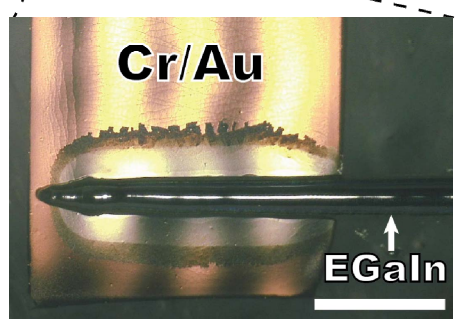

Figure 4: Materials constituting the soft metal flexionsensing strip. a) Cross section of an EGaIn microwire (scale bar: $100 \mu \mathrm{m})$. b) SEM image of the stretchable $5 / 25 \mathrm{~nm} \mathrm{Cr} / \mathrm{Au}$ thin film (scale bar: $1 \mu \mathrm{m}$ ). c) Top view of the interconnection zone between the soft micro-wire and thin film (scale bar: $500 \mu \mathrm{m}$ ).

Each sensor output was read in real time using three voltage dividers connected to the analog inputs of an Arduino Micro board. The board was addressed from a PC through the serial communication bus. We assessed the repeatability and linearity of the sensors by recording the relative increase in resistance of sensor 1 when the MCP joint was incrementally closed multiple times (Figure 6). Small standard deviations and high $\mathrm{R}^{2}$ value indicated good performance of the sensors.

\section{Computation of the joint's angles}

We made the following assumptions in order to compute the three joints angle from the output of the sensors:

- The rotation of each joint resulted in a linear increase of the electrical resistance of each strain gauge.

- This increase in resistance was independent from the position or rotation of the other joints.

Hence, the relative increase in resistance of each sensor was a linear combination of the three joints' angles, with fixed coefficients. We defined the vectors $\boldsymbol{\theta}=\left[\theta_{M C P} ; \theta_{P I P}, \theta_{D I P}\right] \quad$ and $\boldsymbol{R}=\left[\Delta R_{1} / R_{1,0} ; \Delta R_{2} / R_{2,0} ; \Delta R_{3} / R_{3,0}\right]$. We had:

$$
\boldsymbol{R}=J \boldsymbol{\theta}
$$

where $J=K^{-1}$ was a $3 \times 3$ matrix.

The calibration matrix $K(\boldsymbol{\theta}=K \boldsymbol{R})$ was determined with the following calibration scheme. The outputs of the sensors were recorded for six different known positions of the finger and arranged in an $18 \times 9$ matrix $R_{c a l}$. The angles of the three joints for the six known positions were arranged in an eighteen elements vector $\boldsymbol{\theta}_{\text {cal. }}$. The nine
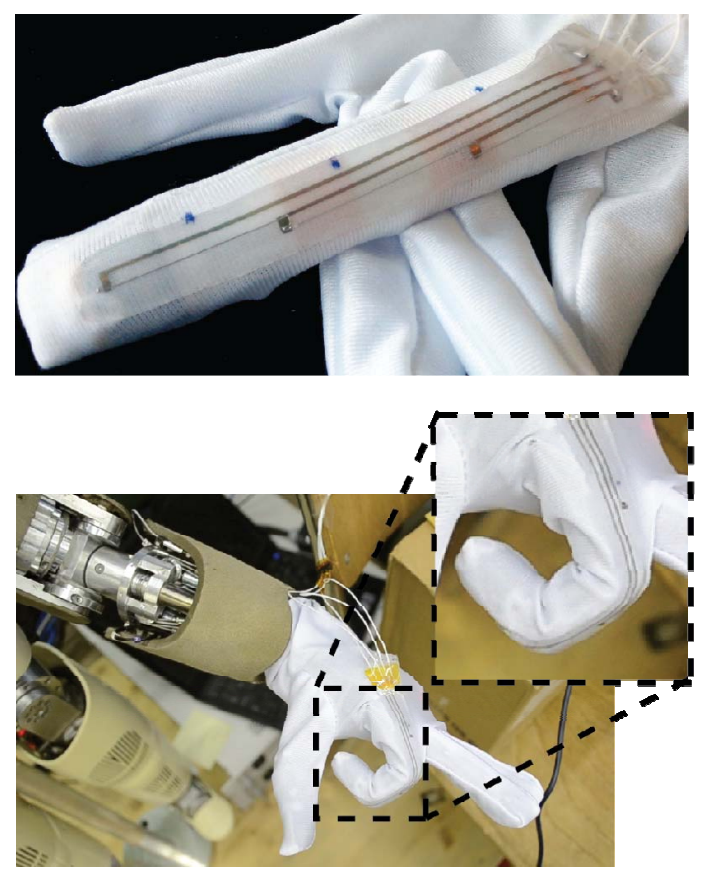

Figure 5: Soft flexion sensing trip after mounting on a textile glove and worn on the iCub robot with its finger fully flexed.

elements vector containing the coefficients of matrix $K$, $\boldsymbol{C}_{\boldsymbol{K}}$, was computed using the least square method:

$$
\boldsymbol{C}_{\boldsymbol{K}}=\left(R_{\text {cal }}{ }^{T} R_{\text {cal }}\right)^{-1} R_{\text {cal }}^{T} \boldsymbol{\theta}_{\text {cal }}
$$

This calibration routine was implemented in Matlab (MathWorks).

\section{Real-time acquisition of the hand posture}

After determination of the calibration matrix $K$, a Matlab script converted the sensors' outputs into joint angles and displayed the finger posture. Figure 8 presents the computed angles and reconstructed finger posture for repeated complete closings of the robot's hand. The MCP joint was first rotated. Then, PIP and DIP joints were completely closed at the same time. The joints were actuated in the inverse order for opening. Data show the

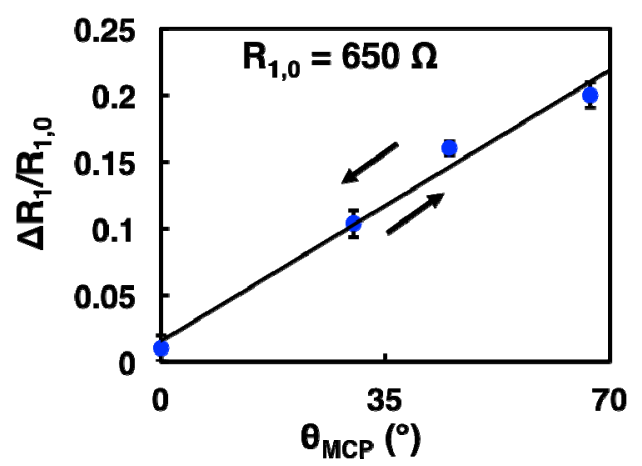

Figure 6: Response of sensor 1 to flexion of the MCP joint. 12 flexion cycles from 0 to $67^{\circ}$ are represented. Error bars represent 99\% confidence interval. Solid line represents linear interpolation $\left(R^{2}=0.98\right)$. 


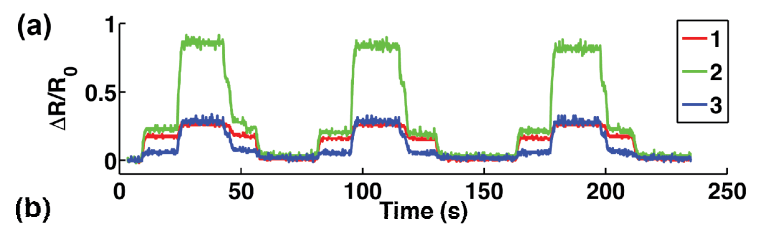

(b)

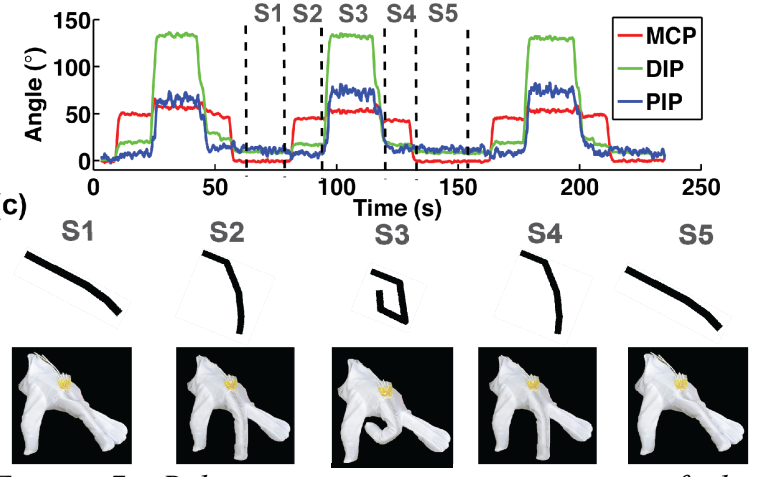

Figure 7: Relative increase in resistance of the sensors and computed joints angles as a function of time when the iCub hand is completely closed three times. Comparison between reconstructed finger profile and actual hand profile.

sensors were stable, quickly adapted to finger motion with good repeatability, and did not overshoot (Figure 7). We also observed a systematic overestimation of $\theta_{P I P}$ and underestimation of $\theta_{D I P}$ when the finger was completely closed. This might come from non-linearity or global strain (not only in the vicinity of the joints) occurring in the skin during finger bending. These phenomena were not taken into account in the calibration scheme. With longer interconnects, the accuracy of the sensors could have been improved by patterning a single strain sensing area per joint, thus mechanically reducing crosstalk between sensors. For example, as sensor 1 was covering the MCP joint only, it was mainly sensitive to the closing of the MCP joint and not the rotation of the other two joints (Figure 7a). However, the calibration scheme was general and could be used for compensating crosstalk in alternative sensor's layout.

\section{CONCLUSION}

We reported the fabrication and characterization of stretchable, skin-like flexion sensors that encoded the relative rotation of finger's joints. Ad-hoc choice of materials and fabrication methods resulted in a thin and fully stretchable system with soft integrated interconnects and strain gauges. We mounted the skin system on the iCub humanoid hand to assess the sensors' linearity, repeatability and dynamic behavior. We also proposed a calibration algorithm that overcame design limitations and enabled reconstruction of the finger posture in real time. Modification of the sensor's design could enhance accuracy of the sensors. Long term response and fatigue resistance of the proposed sensing skin still need to be investigated and quantified.

Future applications could involve development of a data glove for the human hand and coupling with other soft transducers such as tactile sensors.

\section{ACKNOWLEDGEMENTS}

This work was sponsored by the Nanotera.ch initiative within the WiseSkin project. We warmly acknowledge Nicolas Sommer and Prof. Aude Billard from LASA (EPFL) for access and help with the iCub robot.

\section{REFERENCES}

[1] L. Dipietro, A. Sabatini, and P. Dario, "A survey of glove-based systems and their applications," IEEE Trans. Syst., Man, Cybern. C, Appl. Rev., vol. 38, no. 4, pp. 461-482, 2008.

[2] G. D. Mura, F. Lorussi, A. Tognetti, G. Anania, N. Carbonaro, M. Pacelli, R. Paradiso, and D. De Rossi, "Piezoresistive Goniometer Network for Sensing Gloves", in IFMBE Proc. 41, 2014, pp. 1547-1550.

[3] M. L. Hammock, A. Chortos, B. C.-K. Tee, J. B.-H. Tok, and Z. Bao, "The evolution of electronic skin (eskin): a brief history, design considerations, and recent progress.," Adv. Mater., vol. 25, no. 42, pp. 59976038, 2013.

[4] M. Park, J. Park, and U. Jeong, "Design of conductive composite elastomers for stretchable electronics", Nano Today, vol. 9, no. 2, pp. 244-260, 2014.

[5] Q. Zheng, J. F. Zhou, and Y. H. Song, "Timedependent uniaxial piezoresistive behavior of highdensity polyethylene/short carbon fiber conductive composites", J. Mater. Res., vol. 19, no. 9, pp. 26252634, Mar. 2011.

[6] J. T. Muth, D. M. Vogt, R. L. Truby, Y. Mengüç, D. B. Kolesky, R. J. Wood, and J. a. Lewis, "Embedded 3D Printing of Strain Sensors within Highly Stretchable Elastomers," Adv. Mater., vol. 26, pp. 6307-6312, 2014.

[7] F. L. Hammond, Y. Mengüç, and R. J. Wood, "Toward a Modular Soft Sensor - Embedded Glove for Human Hand Motion and Tactile Pressure Measurement," in IROS 2014, 2014, pp. 4000-4007

[8] S. P. Lacour, S. Wagner, Z. Huang, and Z. Suo, "Stretchable gold conductors on elastomeric substrates", Appl. Phys. Lett., vol. 82, no. 15, p. 2404, 2003.

[9] J. W. Boley, E. L. White, G. T.-C. Chiu, and R. K. Kramer, "Direct Writing of Gallium-Indium Alloy for Stretchable Electronics," Adv. Funct. Mater., pp. 3501-3507, Feb. 2014.

[10] A. Schmitz, U. Pattacini, F. Nori, L. Natale, G. Metta, and G. Sandini, "Design, realization and sensorization of a dextrous hand: the $\mathrm{iCub}$ design choices", in Humanoids 2010, 2010, pp. 186-191

[11] H.O. Michaud, J. Teixidor, and S.P. Lacour, "Soft metal constructs for large strain sensor membrane", under revision, 2014.

[12] H.J. Kim, "Stretchable Interconnects Using Room Temperature Liquid Alloy on Elastomeric Substrate", Ph.D. thesis Purdue University, USA, 2007.

\section{CONTACT}

*S.P. Lacour, stephanie.lacour@epfl.ch 